\title{
A Review of miR-326 and Female Related Diseases
}

\author{
Li-na Lin ${ }^{1}$, Qing-mei Zhang ${ }^{1,2}$, Ying-ying Ge ${ }^{1,2}$, Bin Luo ${ }^{1,2}$ and Xiao-xun Xie ${ }^{1,2,3}$ \\ ${ }^{1}$ Department of Histology and Embryology, School of Pre-clinical Medicine, Guangxi Medical University, Nanning, Guangxi \\ Zhuang Autonomous Region, P. R. China, ${ }^{2}$ Key Laboratory Research of Preclinical Medicine of Guangxi Colleges and \\ Universities, Guangxi Medical University, Nanning, Guangxi Zhuang Autonomous Region, P. R. China and ${ }^{3}$ Key Laboratory \\ of Early Prevention and Treatment of Regional High Frequency Tumor (Guangxi Medical University), Ministry of Education, \\ P. R. China
}

Received October 11, 2020; accepted March 7, 2021; published online April 22, 2021

\begin{abstract}
MicroRNA (miRNA), a non-coding single-stranded RNA molecule with 20-23 nucleotides encoded by endogenous genes, plays an essential role in maintaining normal cell function and regulating cell proliferation, differentiation, apoptosis, autophagy, and cell metabolism. The imbalance between miRNA and genes can cause a series of diseases, including malignancies. miRNA-326 (miR-326) is extensively known for its core regulation of various biological processes. This review presents an overview of the highlights of miR-326 in female-related diseases. To understand the impact of miR-326 on female disorders, we search all published studies about miR-326 having a high incidence in female conditions, including cervical cancer, endometrial cancer, breast cancer, intrauterine adhesion, and multiple autoimmune diseases. We aim to learn about the mutual regulation mechanism between miR-326 and related genes and signaling pathways, as well as to elaborate on the value of miR-326 as a potential biomarker and therapeutic target of female diseases. Our results provide reliable evidence and new strategies for treating female tumors and autoimmune diseases.
\end{abstract}

Key words: microRNA, miR-326, tumor, intrauterine adhesion, autoimmune diseases

\section{Introduction}

MicroRNAs (miRNAs) are the most abundant and vital class of gene regulatory factors in organisms. They play essential roles in regulating endogenous genes and in genome resistance to the invasion of exogenous nucleic acids. They are involved in various biological processes, such as individual development, cell differentiation, proliferation, apoptosis, and stress regulation of target genes [4]. The dysregulation of miRNAs reportedly leads to cellular homeostasis disorder. Over the past decade, miRNAs have received extensive attention for their pivotal roles in the occurrence and development of numerous human diseases,

Correspondence to: Xiao-xun Xie, PhD, Professor of School of Preclinical Medicine, Guangxi Medical University, 22 Shuangyong Road, Nanning, China.

E-mail: 1695261181@qq.com and xiexiaoxun@gxmu.edu.cn particularly cancers and autoimmune diseases (ADs). Considering that miRNAs can control multiple genes in different cancers or illnesses, they are also considered potential therapeutic targets or diagnostic biomarkers. However, their exact roles and molecular mechanisms have not yet been fully defined. miRNA-326 (miR-326), as a tumor suppressor, participates in the tumorigenesis, invasion, and metastasis of multiple cancers, such as cervical cancer (CC), endometrial cancer, and breast cancer (BC) [45]. Given that miR-326 is related to immune regulation, its role in ADs is gradually attracting research attention. The majority of miR-326-related diseases present a high incidence in females. In this Review, we discuss the roles of miR-326 in female-related diseases to further understand its regulatory mechanism in these diseases. We also provide strong evidence for miR-326 as an ideal biomarker, therapeutic target, and prognostic indicator in many female-related diseases. 


\section{II. miRNAs}

Mature miRNAs (20-23 nucleotides long) function as post-transcriptional gene regulators that regulate gene expression by combining with the 3 '-untranslated region (3'-UTR) of their target genes. Consequently, transcripts are degraded and/or translation is inhibited [5]. Initially, miRNAs are transcribed by RNA polymerase II from the genome into primary transcripts (pri-miRNAs). PrimiRNAs are then cleaved in the nucleus by a microprocessing complex Drosha-DGCR8 into a 60-70 nt stem loop precursor (pre-miRNAs) before being exported into the cytoplasm by nuclear transport receptor Exportin-5-RanGTP. In the cytoplasm, pre-miRNAs are further cleaved into mature length by Dicer. Finally, the double-stranded miRNA is recruited by Argonaute proteins and incorporated into the RNA-induced silencing complex (RISC). One strand of miRNA double strand is preserved in the RISC complex, whereas the other strand is excreted from the complex and rapidly degraded. Mature miRNAs repress the mRNA translation or destabilize mRNA transcripts, regulating protein expression levels [20, 30, 33] (Fig. 1). Currently, more than 2500 miRNAs have been identified. They can regulate the expression of one-third of all genes in the human body. Since they have been identified, considerable studies have confirmed that miRNAs participate in various human tumors and other diseases. Many miRNAs have varying degrees of diversity in sequence, structure, expression, and function. The discovery of miRNAs have led to a worldwide research effort to explore their roles in cancers and other diseases. Aberrantly expressed miRNAs are reportedly correlated with tumorigenesis, tumor progression, and metastasis. Some miRNAs have been verified to function as oncogenes (onco-miRNAs), such as miR-21, miR-23, miR-107, miR-155, miR-210, miR-221, miR-17 92 cluster, and miR-10b. Conversely, some function as a tumor suppressor (suppressor miRNA), such as miR-34 family, let-7 family, miR-200 family, miR-15/16, miR-375, miR-506, and miR-520, depending on their targeting genes and tumor types [29, 51]. These oncomiRNAs or suppressor miRNAs form a complex biological regulatory network through their target genes, upstream transcription factors, and various feedback regulatory circuits to participate in tumor development. miRNAs are essential for multiple cellular homeostases, so deregulation between miRNAs and target genes leads to tumorigenesis. Studies have demonstrated that miRNAs play an important role in proliferation, differentiation, autonomous growth signals, apoptosis, angiogenesis, invasion, metastasis, energy metabolism, immune escape, and tumor resistance [18]. Some miRNAs play an important role in embryo implantation, cell growth, differentiation, tissue homogeneity and organ formation during embryonic development. They affect many different developmental processes, such as osteogenic and chondrogenic differentiation, neuronal and cardiac differentiation, endothelial differentiation and

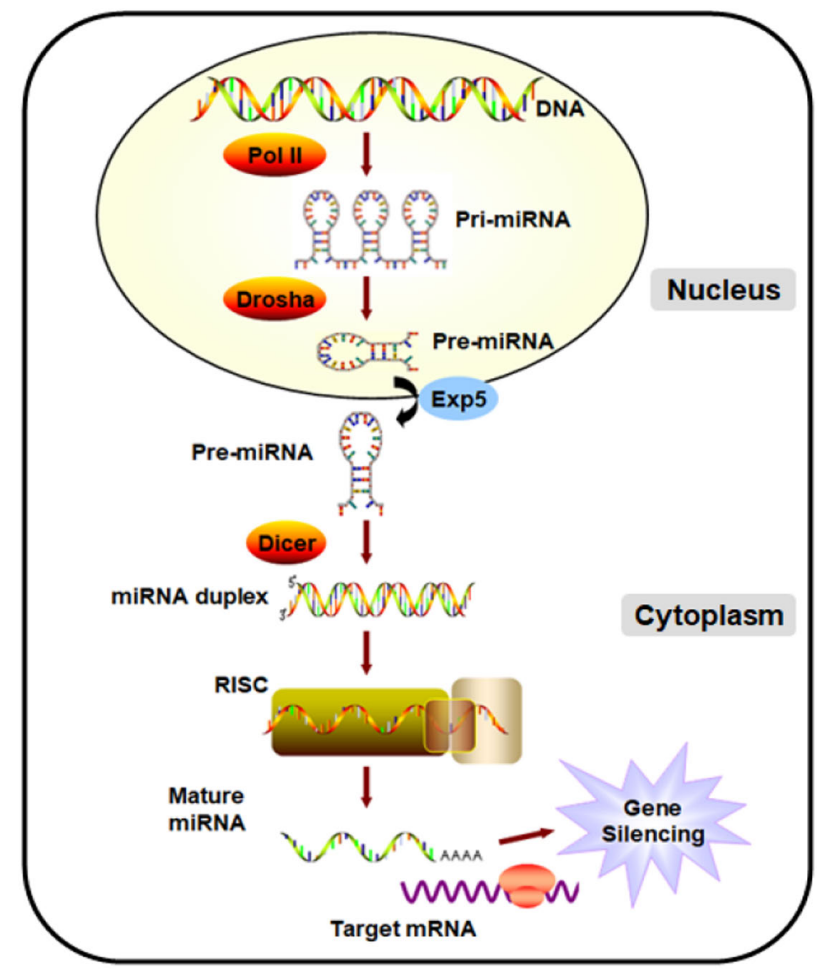

Fig. 1. miRNA biogenesis. Schematic representation of miRNA biogenesis and function.

hematopoiesis. In recent years, the regulation of miRNAs on glucose metabolism and lipid metabolism has become a hot spot in metabolic diseases research [52, 62]. miRNAs also perform a crucial regulatory function in various physiological and pathological processes such as inflammation, immune response, hematopoiesis, embryonic development, and organ development, thereby affecting multiple human disorders (e.g., ADs, cardiovascular diseases, diabetes, hepatitis, and neurological disorders). Hence, miRNAs may serve as potential biomarkers and therapeutic targets and should be extensively explored in various cancers and diseases.

\section{III. miR-326}

In 2004, miR-326 was identified as a neural-specific miRNA in neurons [42]. The precursor gene of miR-326 is located within chromosome 11 in intron 1 of the betaarrestin gene (ARRB1) [39]. miR-326 belongs to the miR-15/107 gene group, and its expression is associated with brain development and neural stem-cell differentiation $[7,27]$. It serves as a tumor suppressor and has gained extensive research attention in recent years. miR-326 overexpression in various human cancers has been confirmed. It is involved in the development and progression of tumors by regulating cell growth, apoptosis, migration, and invasion [3]. miR-326 can be detected in patients' peripheral blood with $\mathrm{ADs}$, so it is proposed to regulate T-cell differ- 
entiation and mediate the immune response. Studies are increasingly focusing on the clinical benefit of miR-326 in ADs and autoinflammation (e.g., multiple sclerosis (MS), systemic lupus erythematosus (SLE), immune thrombocytopenia (ITP), and type-1 diabetes mellitus (T1DM)) [21, 54]. Thus, miR-326 is considered as an immune-related miRNA. miR-326 is also reportedly related to some fibrotic diseases, such as idiopathic pulmonary fibrosis, liver fibrosis, and intrauterine adhesion (IUA) [70]. Among the numerous conditions related to miR-326, a considerable part is more predominant in women. Here, we review the role of miR-326 in female-related diseases, providing evidence that miR-326 is a potential biomarker and therapeutic target for such diseases, including cancers.

\section{IV. miR-326 in Female-related Cancers}

\section{Cervical cancer (CC)}

$\mathrm{CC}$ is the fourth most common cancer and the second leading cause of cancer-related mortality in females worldwide [57]. Surgery combined with radiation or chemotherapy is the first-line therapeutic approach to treating CC. However, metastasis and recurrence remain the leading causes of high mortality in advanced patients. Over the past decade, various miRNAs have been proven to be abnormally expressed in CC and contribute to CC's pathological progress by acting either as oncogenes or tumor suppressors [46]. Studies have shown that miR-326 is downregulated in $\mathrm{CC}$ tissues and cell lines. Zhang et al. demonstrated that miR-326 is significantly downregulated in CC tissues compared with healthy control specimens. miR-326 overexpression inhibits the proliferation, migration, and invasion of $\mathrm{CC}$ cell lines by targeting ETS domain-containing protein Elk-1 (ELK1). ELK1 is a transcription factor of the MAPK signaling pathway that directly regulates cytoskeleton-associated activities and cell migration. Moreover, ELK1 could mitigate the suppressive effects on proliferation, migration, and invasion exerted by miR-326 [67]. These findings have been confirmed by another study involving 63 patients and 40 normal cases. Results show that miR-326 is significantly downregulated in the CC group, whereas a circular RNA (circRNA) hsa_circ_0000515 and ELK1 are upregulated. Given that miR-326 can directly target hsa_circ_0000515 and ELK1, hsa_circ_0000515 competitively binds to miR-326 and acts as a sponge to regulate ELK1 expression. Eventually, the proliferation and invasion of $\mathrm{CC}$ cells are enhanced, and their apoptosis and autophagy are reduced [56]. Cheng et al. detected miR-326 in 12 cases of cervical intraepithelial neoplasia (CIN) and 24 cases of squamous carcinoma and discovered gradually elevated miR-326 from CIN to squamous carcinoma. miR-326 reflects the progression from precancerous lesions to invasive cancers, rendering it as a potential CC diagnosis predictor. They also observed that vascular endothelial growth factor C (VEGF-C) promotes $\mathrm{CC}$ cell invasiveness by suppressing miR-326 through the
c-Src pathway. Perhaps the miR-326 genetic locus is in the first intron of the host gene ARRB1, and c-Src can regulate transcription factors of ARRB1 gene, thereby affecting miR-326 expression [6]. Further studies should be conducted to validate this supposition. Recently, miR-326 has been reported as a target of long non-coding RNA TDRG1, which promotes the proliferation, migration, and invasion of $\mathrm{CC}$ cells by targeting miR-326 to regulate MAPK1 expression. Besides, knockdown of miR-326 can reverse the reduced apoptosis caused by TDRG1 downregulation [22]. The above studies show that miR-326 plays a role in inhibiting the proliferation, invasion and metastasis of $\mathrm{CC}$, but the specific mechanism has not been elucidated.

\section{Endometrial carcinoma (EC)}

EC is a common type of gynecological cancer. EC has two types, and the outcome of Type II is poorer than that of Type I because Type II EC is estrogen independent. This feature means that it is characterized by poor differentiation, high malignancy, negative estrogen and progesterone receptor, and insensitivity to chemotherapy. Studies have shown that miR-326 affects the malignant behaviors of EC cells and promotes tumor progression. Gao et al. reported that miR-326 could target the G proteincoupled receptor 91 (GPR91), known as succinate receptor 1. GPR91 participates in several metabolic pathways. Gao and his colleagues used superparamagnetic iron oxide nanoparticle (SPION) to mediate the efficient miR-326 expression(miR-326@SPION). They found that miR-326 overexpression inhibits the proliferation, division, invasion, and tumorigenicity of EC stem cells in vitro and in vivo by activating the GPR91/STAT3/VEGF signaling pathway [15]. miR-326 is also remarkably downregulated in EC tissues and cell lines as demonstrated by Liu et al. study. They verified that transcription factor TWIST1 is another target of miR-326. The upregulation of miR-326 inhibits EC cell migration by suppressing epithelial-mesenchymal transition (EMT) via TWIST1 [35]. EMT is well known to be an essential mechanism of tumor metastasis. Previous studies have also suggested that mir-326 mimic can prevent epithelial cells from transforming into mesenchymal cells by targeting transforming growth factor- $\beta$ (TGF- $\beta$ ) [9]. All these findings indicate that miR-326 may be a promising metastasis suppressor in EC.

\section{Breast cancer (BC)}

$\mathrm{BC}$ is the most common malignancy in women worldwide and seriously threats women's health and lives. Chemotherapy resistances are urgent problems to be solved. Multidrug resistance (MDR) is one of the main issues in chemoresistance. Liang et al. demonstrated for the first time that miR-326 could mediate the MDR-associated protein (MRP-1/ABCC1) to improve the sensitivity of $\mathrm{BC}$ cells to chemotherapy drugs. Upon transfecting etoposide (VP-16)-resistant cells with miR-326 mimic, MRP-1/ $\mathrm{ABCC} 1$ expression decreases, and cell sensitivity to VP-16 
and doxorubicin increases [31]. Another study has confirmed these findings. Baldassari et al. found that miR-326 can significantly enhance some specific BC drug activity by combining treatment, such as gefitinib, erlotinib, LEE011, BYL719, AZD5363, and SCH772984 [2]. However, the particular mechanism and potential drug targets require further investigation. Similarly, miR-326 acts as a tumor suppressor participating in BC progression. Interestingly, the sex-determining region Y-box protein 12 (SOX12), a known oncogene, has been identified as a target of miR-326; thus, targeting SOX12 by miR-326 can inhibit BC development and progression [12]. Pan et al. explored the expression profile of miR-326 in $\mathrm{BC}$ tissues and found that it expresses significantly lower miR-326 than para-carcinoma tissue and other healthy tissues. miR-326 can also be regulated by a circRNA, such as circ 0061825 (circ-TFF1), whose knockdown can promote miR-326 expression in BC cells. Moreover, circTFF1 serves as a competing endogenous RNA of TFF1 by sponging miR-326, promoting the migration, invasion, and EMT of BC cells in vitro and in vivo [44]. Du et al. investigated the effect of miR-326 on cell cycle and showed that miR-326 restrains $\mathrm{BC}$ cell proliferation by regulating cell-cycle arrest at G1/G0 stage [12]. According to Ghaemi et al., miR-326 suppresses BC cell proliferation. It promotes the apoptosis and upregulation of miR-326, leading to increased sub-G1 cell proportion and decreased $\mathrm{G} 0 / \mathrm{G} 1, \mathrm{~S}$, and G2/M cell proportions. By targeting ErbB2, ErbB3, and AKT, miR-326 inhibits the PI3K/AKT signaling pathway and affects the malignant biological behaviors of BC cells [16].

Taken together, the above results indicate that miR-326 may serve as a tumor suppressor. It is involved in many signaling pathways, participates in the regulation of tumor-cell biological processes by acting as a target or targeting related genes, and affects cell proliferation, apoptosis, migration, invasion, and drug resistance in various female cancers. However, the interaction mechanisms between miR-326 and target genes require further exploration.

\section{V. miR-326 in Intrauterine Adhesion (IUA)}

IUA is essentially a fibrotic endometrial disease caused by the deposition of collagen fibers in the uterine owing to severe damage and repair of the endometrium. IUA, also known as Ashermaii syndrome, can lead to hypomenorrhea, amenorrhea, secondary sterility, and recurrent pregnancy loss, extremely affecting women's health and fertility $[13,64]$. At present, IUA pathogenesis lacks systematic understanding. No ideal therapeutic strategy has been established to date. miR-326 can regulate the expression of fibrosis-related genes such as MMP-9 and Smad7, so it participates in the pathogenesis of pulmonary fibrosis [9]. Ning et al. proposed the involvement of miR-326 in IUA. They found that miR-326 expression in endometrial tissues of IUA is lower than that of normal endometrial tissues and negatively correlated with the expression of fibrotic markers (TGF- $\beta 1, \alpha$-SMA, COL1A1, and FN). Furthermore, a luciferase reporter assay has proven that TGF- $\beta 1$ is a direct target of miR-326. TGF- $\beta$, as the most prominent factor in EMT, is involved in organ fibrosis. This finding suggests that miR-326 could protect endometrial stromal cells from fibrosis by negatively regulating the TGF- $\beta 1 / \mathrm{Smad} 3$ pathway [40]. Additionally, miR-326 is associated with the pathogenesis of pulmonary fibrosis and liver fibrosis $[32,60]$. Multiple studies have shown that the $\mathrm{NF}-\kappa \mathrm{B}$, TGF- $\beta 1 / \mathrm{Smad}$, and $\mathrm{wnt} / \beta$-catenin signaling pathways are classic pathways related to endometrial fibrosis and IUA $[10,37,61]$. miR-326 may affect the process of endometrial fibrosis by regulating central factors affecting these pathways. Therefore, more work is needed to investigate the potential role of miR-326 in IUA treatment.

\section{VI. miR-326 in Female-related Autoimmunity Disease}

ADs are a type of disorders characterized by the body's immune response to autoantibody resulting in damage to its tissues or organs. Women have a high incidence of up to $80 \%$ among $\mathrm{AD}$ patients [50]. Genetic factors and environmental factors are involved in AD pathogenesis, but the exact pathogenesis is presently unclear. In the miRNAs family, miR-326 is one of the research hotspots, and its role in ADs has gradually attracted attention. Many studies have shown that miR-326 is abnormally expressed in various ADs.

\section{Systemic lupus erythematosus (SLE)}

SLE is a typical chronic AD involving multiple organs and systems. SLE is more common in women of childbearing age and leads to adverse pregnancy outcomes, such as abortion, premature delivery, and intrauterine growth retardation. Abnormal autoimmune tolerance plays a vital role in SLE pathogenesis, especially T-cell immune disorders. Regulatory $\mathrm{T}$ cells (Treg) plays an essential role in regulating the development of various ADs, including SLE [43, 68]. Interleukin (IL) 17-producing T helper (Th17) cells are a new type of immune effector cells different from Th1/Th2 cells, which primarily mediate the occurrence of $A D$ and chronic inflammation by promoting the secretion of inflammatory cytokines, such as IL-17, IL-21, and IL-22 [38]. A previous research has proposed that Th17 cells play a crucial role in most occurrences of $\mathrm{AD}$ and their development [25]. Sun et al. found that miR-326 expression in Treg cells in peripheral blood from patients with primary SLE abnormally increases and is proportional to the disease severity. Simultaneously, the level of E26-transformation-specifc-1 (Ets-1) is negatively correlated with miR-326 in SLE. Ets-1 is a transcription factor with an essential part in regulating Th17 differentiation and immune responses. These data suggest that miR-326 may participate in the occurrence and 
activity of SLE by inhibiting Ets-1 expression [55]. Dai et al. detected the mRNA expression of miR-326 and Ets-1 and calculated the ratio of Thl7 cells to CD4+ T cells of 40 patients with SLE. They also analyzed the expression levels of miR-326 and ETS-1 and the positive rate of Th17 cells association with the severity of lupus activity, system involvement, and clinical indicators. They found that miR-326 is involved in the pathogenesis of SLE by regulating Ets- 1 expression and Th17 cell differentiation; it is also closely related to the clinical phenotype of SLE [8]. A study has demonstrated that miR-326 could regulate B-cell differentiation by enhancing B-cell hyperactivity via Ets-1 and promoting autoantibody production in the lupus disease of an animal module (MRL/lpr mice) [23, 59].

\section{Multiple sclerosis (MS)}

MS is an inflammatory AD of the central nervous system characterized by brain and spinal-cord demyelination. In recent years, Th17 cells and Treg cells have been found to play an important role in MS development. Du et al. found that miR-326 expression is positively correlated with Th17 cells in peripheral blood lymphocytes of MS patients, and that CD4+ $\mathrm{T}$ cells are co-expressed with miR-326 and IL-17A considered as a pro-inflammatory cytokine secreted by Th17 cell. Further experiments show that by silencing or overexpressing miR-326 in the mice with experimental autoimmune encephalomyelitis, the number of Th17 cells decreases or increases accordingly [11]. A study has demonstrated that miR-326 could bind to Ets-1, which functions as a Th17 negative transcription factor and prevents Ets-1 translation, eventually promoting Th17 cell differentiation and inflammatory response [64]. Junker and his colleagues discovered that miR-326 is upregulated in active MS lesions and targets the 3'-UTR of CD47, thereby reducing CD47. The importance of CD47 is reflected by its inhibitory role for macrophages whose activity is closely related to MS progression [24]. Similarly, Honardoost et al. demonstrated upregulated miR-326 expression in 40 relapsing-remitting MS (RR-MS) patients compared with patients in the remission phase and healthy individuals. Their results further suggest that miR-326 enhances Th17 cell differentiation during MS development by targeting key factors (Smad1 and Smad4) in TGF- $\beta$ signaling [19]. Several studies have confirmed that miR-326 is highly expressed in the peripheral blood mononuclear cells (PBMC) of RR-MS patients compared with healthy controls $[58,66]$. However, the specific molecular mechanisms remain unclear. Niwald et al. observed elevated miR-326 in sera of $72 \%$ of RR-MS patients, but no statistically significant differences exist between post-acute and stable remission phases [41].

\section{Autoimmune thyroiditis}

Autoimmune thyroid disease is a group of organspecific ADS caused by the exposure and activation of thyroid autoantigens owing to autoimmune disorder, including
Graves' disease and autoimmune thyroiditis (AIT). AIT is characterized by lymphocyte infiltration and follicular destruction of the thyroid [47]. Thyroid-specific antibodies (TPOAb and $\mathrm{TgAb}$ ) significantly increase in the serum, and high levels of TPOAb and TgAb are mostly present in female populations. AIT risk is higher among women than men [49]. Patients with AIT are often accompanied by other ADs, such as MS, T1DM, and rheumatoid arthritis. Th17 cell differentiation and maturation are reportedly associated with numerous cytokines and other factors, including miR-326 [14, 53, 63]. Zhao et al. investigated 55 AIT patients and 23 healthy volunteers. They found that the expression levels of miR-326 and Th17 in PBMC and thyroid tissue of AIT patients are higher than those in healthy controls, and the expression levels of miR-326 and Th17 are significantly negatively correlated with Ets-1 expression. They further demonstrated that the inhibition of miR-326 results in decreased inflammation and downregulation of the expression levels of Th17 cell-related cytokines (IL-17a, IL-22) and transcription factors (Rorgt, Rora, and Irf4). However, reduced miR-326 expression upregulates the protein expression of Ets-1, disintegrin and metalloproteinase-17 (ADAM-17) by using a mouse model of AIT (NOD.H-2h4). These data further confirm that miR-326 could affect the differentiation of Th17 cells and participate in the inflammatory response of AIT by targeting the regulation of Ets-1 and ADAM17 [69]. Recently, Liu et al. conducted a study involving 58 Hashimoto's thyroiditis (HT) patients and 55 normal controls. They found that compared with healthy controls, miR-326 expression in PBMCs of HT patients is significantly upregulated. After miR-326 overexpression or inhibition in PBMC in vitro, ADAM-17 expression significantly decreases or increases, respectively. Thus, miR-326 exerts a negative regulatory effect on ADAM-17. ADAM-17 is proven to be a target of miR-326 and affects the differentiation and maturation of Th17 cell by blocking IL-23/IL-23R/pSTAT3 signaling pathway. In brief, miR-326 could regulate the IL-23/ IL-23R/Th17 cell axis in HT and promote disease progression by targeting ADAM17 [36].

\section{Immune thrombocytopenia (ITP)}

ITP is an acquired AD characterized by accelerated platelet destruction and decreased peripheral platelet count [28]. It is common in women of childbearing age. Although the antiplatelet antibody resulting in overdestruction of platelets may be prominent in ITP pathophysiology, Tlymphocyte-mediated immune disturbance may also play an important role in its pathogenesis and disease progression. At present, literature about miR-326 in ITP is scant. Yu et al. showed that miR-326 transfected into leukocytedepleted apheresis platelets can promote platelet apoptosis by targeting the anti-apoptotic Bcl-xL gene, but it could not induce platelet activation [65]. Moreover, Liu et al. investigated the expression of a series of miRNAs (miR-326, miR-181a, miR-146a, and miR-142-3p) in PBMCs of 
patients with ITP. They found that three of miRNAs including miR-326 significantly decrease compared with the control group. Hence, miR-326 acts as an immune-related microRNA, playing an essential role in ITP pathogenesis [34]. These results provide a basis for an in-depth understanding of the regulation mechanism of miR326 in TIP.

\section{Diabetes type 1 (T1DM)}

T1DM is an AD that occurs in individuals with a genetic predisposition under the combined action of genetic and environmental factors. The destruction of islet $\beta$ cells and the inflammation of islet cells are its main pathological features, and these phenomena lead to insufficient insulin secretion and eventual development into diabetes. Sebastiani et al. indicated that miR-326 expression is abnormally elevated in T1DM patients and nearly related to the expression level of autoantibodies (GADA and/or IA-2A) in peripheral blood. miR-326 expression level has also been suggested to reflect disease severity [53]. Epidemiological studies have confirmed that vitamin $\mathrm{D}$ is significantly related to T1DM. miR-326 upregulation can target VDR and inhibit its expression in immune cells, enhance T cell activity, and induce T1DM [17, 26, 48]. Azhir et al. also found that miR-326 is over-expressed in patients with T1DM compared with healthy individuals. Interestingly, their results show that miR-326 expression is higher in patients with age at disease onset of below 14 years old than in those with age at disease onset of more than 14 years old. Patients with 5-10 years of disease duration also present higher miR-326 expression. Besides, Azhir et al. confirmed that multiple genes (TBX21, CD69, CYCS, and IKZF2) are specific targets of miR-326 in T1DM [1]. These data provide new therapeutic targets for autoimmune diabetes. A study on the role and mechanism of miR-326 targeting the negative regulation of $\operatorname{IgG}$ Fc $\gamma$ RIII in the immune damage of diabetic nephropathy is ongoing. With the development of mechanism research, we may discover more value of miR-326 in treating immune diseases.

In summary, miR-326 is abnormally expressed in various ADs. By regulating its target genes, miR-326 affects various cytokines or transcription factors, promotes lymphocyte differentiation, and participates in the occurrence and development of ADs. However, the occurrence of $\mathrm{AD}$ is the result of a combination of multiple factors. In different ADs, miR-326 has different targets and immunesystem regulation mechanisms, thereby requiring further future investigations.

\section{Conclusions}

We discuss the involvement of miR-326 in femalerelated cancers and ADs, including related genes and significant signaling pathways. miR-326 may serve as a tumor suppressor by regulating target genes and pathways. It may also play a crucial role in promoting lymphocyte dif- ferentiation and the pathogenesis of many common ADs. Targeting miR-326 or combining traditional therapy with miR-326 mimics may be accepted as a new treatment strategy in these autoimmune and malignant diseases. Moreover, given that miR-326 expression is closely associated with tumor prognosis and disease state, it can also act as a useful prognostic indicator. However, more work is needed to explore the concrete regulation mechanisms between miR-326 and these diseases.

\section{Conflicts of Interest} of interest.

The authors declare that there are no conflicts

\section{Acknowledgments}

This work was supported by the National Natural Science Foundation of China (No. 81960453, No. 81860445 and No. 81660429), Natural Science Foundation of Guangxi Province (No. 2018GXNSFAA050151, No. 2018GXNSFAA281050, No. 2018GXNSFAA281251, No. 2017GXNSFAA198001, No. 2018GXNSFAA050058 and No. 2018GXNSFBA281187), Key Laboratory of Early Prevention and Treatment for Regional High Frequency Tumor (Guangxi Medical University) and Ministry of Education (No. GK2018-09, No. GKE 2019-08, and No. K2015TKF03), and Guangxi First-class Discipline Construction Project in Basic Medical Sciences (No. GXMUBMSTCT07, No. GXMUBMSTCF-G04, No. GXMUBMSTCFG06 and No. GXMUBMSTCF-G11).

\section{References}

1. Azhir, Z., Dehghanian, F. and Hojati, Z. (2018) Increased expression of microRNAs, miR-20a and miR-326 in PBMCs of patients with type 1 diabetes. Mol. Biol. Rep. 45; 1973-1980.

2. Baldassari, F., Zerbinati, C., Galasso, M., Corrà, F., Minotti, L., Agnoletto, C., et al. (2018) Screen for MicroRNA and Drug Interactions in Breast Cancer Cell Lines Points to miR-126 as a Modulator of CDK4/6 and PIK3CA Inhibitors. Front. Genet. 9; 174 .

3. Bartel, D. P. (2004) MicroRNAs: Genomics, biogenesis, mechanism, and function. Cell 116; 281-297.

4. Bushati, N. and Cohen, S. M. (2007) MicroRNA functions. Annu. Rev. Cell Dev. Biol. 23; 175-205.

5. Catalanotto, C., Cogoni, C. and Zardo, G. (2016) MicroRNA in Control of Gene Expression: An Overview of Nuclear Functions. Int. J. Mol. Sci. 17; 1712.

6. Cheng, Y., Jiang, S., Yuan, J., Liu, J. and Simoncini, T. (2018) Vascular endothelial growth factor $\mathrm{C}$ promotes cervical cancer cell invasiveness via regulation of microRNA-326/cortactin expression. Gynecol. Endocrinol. 34; 853-858.

7. Choi, I., Woo, J. H., Jou, I. and Joe, E. H. (2016) PINK1 Deficiency Decreases Expression Levels of mir-326, mir-330, and mir-3099 during Brain Development and Neural Stem Cell Differentiation. Exp. Neurobiol. 25; 14-23.

8. Dai, C., Tao, J. and Sun, X. (2016) miR326 participates 
in SLE morbidity by regulating Ets- 1 expression and Th17 cells differentiation. Acta Universitatis Medicinalis Anhui 51; $1320-1324$

9. Das, S., Kumar, M., Negi, V., Pattnaik, B., Prakash, Y. S., Agrawal, A., et al. (2014) MicroRNA-326 regulates profibrotic functions of transforming growth factor- $\beta$ in pulmonary fibrosis. Am. J. Respir. Cell Mol. Biol. 50; 882-892.

10. Derynck, R. and Zhang, Y. E. (2003) Smad-dependent and Smad-independent pathways in TGF-beta family signalling. Nature 425; 577-584.

11. Du, C., Liu, C., Kang, J., Zhao, G., Ye, Z., Huang, S., et al. (2009) MicroRNA miR-326 regulates TH-17 differentiation and is associated with the pathogenesis of multiple sclerosis. Nat. Immunol. 10; 1252-1259.

12. Du, Y., Shen, L., Zhang, W., Ding, R., Li, Q., Li, S., et al. (2019) Functional analyses of microRNA-326 in breast cancer development. Biosci. Rep. 39; BSR20190787.

13. Evans-Hoeker, E. A. and Young, S. L. (2014) Endometrial receptivity and intrauterine adhesive disease. Semin. Reprod. Med. 32; 392-401.

14. Fallahi, P., Ferrari, S. M., Ruffilli, I., Elia, G., Biricotti, M., Vita, R., et al. (2016) The association of other autoimmune diseases in patients with autoimmune thyroiditis: Review of the literature and report of a large series of patients. Autoimmun. Rev. 15; $1125-1128$.

15. Gao, Y., Qian, H., Tang, X., Du, X., Wang, G., Zhang, H., et al. (2019) Superparamagnetic iron oxide nanoparticle-mediated expression of miR-326 inhibits human endometrial carcinoma stem cell growth. Int. J. Nanomedicine 14; 2719-2731.

16. Ghaemi, Z., Soltani, B. M. and Mowla, S. J. (2019) MicroRNA-326 Functions as a Tumor Suppressor in Breast Cancer by Targeting ErbB/PI3K Signaling PathwayImage. Front. Oncol. 9; 653 .

17. Gregoriou, E., Mamais, I., Tzanetakou, I., Lavranos, G. and Chrysostomou, S. (2017) The effects of vitamin D supplementation in newly diagnosed type 1 diabetes patients: Systematic review of randomized controlled trials. Rev. Diabet. Stud. 14; 260-268.

18. Hayes, J., Peruzzi, P. P. and Lawler, S. (2014) MicroRNAs in cancer: biomarkers, functions and therapy. Trends Mol. Med. 20; 460-469.

19. Honardoost, M. A., Kiani-Esfahani, A., Ghaedi, K., Etemadifar, M. and Salehi, M. (2014) miR-326 and miR-26a, two potential markers for diagnosis of relapse and remission phases in patient with relapsing-remitting multiple sclerosis. Gene 544; 128-133.

20. Ivey, K. N. and Srivastava, D. (2015) microRNAs as Developmental Regulators. Cold Spring Harb. Perspect. Biol. 7; a008144.

21. Jadideslam, G., Ansarin, K., Sakhinia, E., Alipour, S., Pouremamali, F. and Khabbazi, A. (2018) The MicroRNA-326: Autoimmune diseases, diagnostic biomarker, and therapeutic target. J. Cell. Physiol. 233; 9209-9222.

22. Jiang, H., Liang, M., Jiang, Y., Zhang T., Mo, K., Su, S., et al. (2019) The lncRNA TDRG1 promotes cell proliferation, migration and invasion by targeting miR-326 to regulate MAPK1 expression in cervical cancer. Cancer Cell Int. 19; 152.

23. Jin, L., Fang, X., Dai, C., Xiang, N., Sun, X., Azmi, F., et al. (2019) The potential role of Ets-1 and miR-326 in CD19+B cells in the pathogenesis of patients with systemic lupus erythematosus. Clin. Rheumatol. 38; 1031-1038.

24. Junker, A., Krumbholz, M., Eisele, S., Mohan, H., Augstein, F., Bittner, R., et al. (2009) MicroRNA profiling of multiple sclerosis lesions identifies modulators of the regulatory protein CD47. Brain 132; 3342-3352.

25. Kamali, A. N., Noorbakhsh, S. M., Hamedifar, H., Jadidi-
Niaragh, F., Yazdani, R., Bautista, J. M., et al. (2019) A role for Th1-like Th17 cells in the pathogenesis of inflammatory and autoimmune disorders. Mol. Immunol. 105; 107-115.

26. Kamel, M. M., Fouad, S. A., Salaheldin, O., El-Razek Ael-R. and El-Fatah, A. I. (2014) Impact of vitamin D receptor gene polymorphisms in pathogenesis of Type-1 diabetes mellitus. Int . J. Clin. Exp. Med. 7; 5505-5510.

27. Kim, Y., Kim, H., Park, H., Park, D., Lee, H., Lee, Y. S., et al. (2014) FmiR-326-histone deacetylase-3 feedback loop regulates the invasion and tumorigenic and angiogenic response to anticancer drugs. J. Biol. Chem. 289; 28019-28039.

28. Kistangari, G. and McCrae, K. R. (2013) Immune thrombocytopenia. Hematol. Oncol. Clin. North Am. 27; 495-520.

29. Kong, Y., Ferland-Mccollough, D., Jackson, T. J. and Bushell, M. (2012) MicroRNAs in cancer management. Lancet Oncol. 13; e249-58.

30. Liang, Y. (2010) Light Microscopic Radioautographic Study on RNA Synthesis in the Adrenal Glands of Aging Mice. Acta Histochem. Cytochem. 31; 203-210.

31. Liang, Z., Wu, H., Xia, J., Li, Y., Zhang, Y., Huang, $\mathrm{K}$, et al. (2010) Involvement of miR-326 in chemotherapy resistance of breast cancer through modulating expression of multidrug resistance-associated protein 1. Biochem. Pharmacol. 79; 817-824.

32. Liao, X., Zhan, W., Tian, T., Yu, L., Li, R. and Yang, Q. (2019) MicroRNA-326 attenuates hepatic stellate cell activation and liver fibrosis by inhibiting TLR4 signaling. J. Cell. Biochem. $121 ; 3794-3803$.

33. Lipardi, C., Wei, Q. and Paterson, B. M. (2003) RNA silencing in Drosophila. Acta Histochem. Cytochem. 36(2); 123-134.

34. Liu, L., Hua, M., Liu, C., He, N., Li, Z. and Ma, D. (2016) The aberrant expression of microRNAs and correlations with $\mathrm{T}$ cell subsets in patients with immune thrombocytopenia. Oncotarget 7; 76453-76463.

35. Liu, W., Zhang, B., Xu, N., Wang, M. and Liu, Q. (2017) miR-326 regulates EMT and metastasis of endometrial cancer through targeting TWIST1. Eur. Rev. Med. Pharmacol. Sci. 21; 3787-3793.

36. Liu, Y., Cui, X., Wang, S., Liu, J., Zhao, N., Huang, M., et al. (2020) Elevated microRNA-326 levels regulate the IL-23/ IL-23R/Th17 cell axis in Hashimoto's thyroiditis by targeting a disintegrin and metalloprotease 17 . Thyroid 30; 1327-1337. doi: $10.1089 /$ thy.2019.0552.

37. Matsuzaki, S. and Darcha, C. (2013) Involvement of the Wnt/ $\beta$-Catenin Signaling Pathway in the Cellular and Molecular Mechanisms of Fibrosis in Endometriosis. PLoS One 8; e76808.

38. Nalbant, A. (2019) IL-17, IL-21, and IL-22 Cytokines of T Helper 17 Cells in Cancer. J. Interferon Cytokine Res. 39; 56-60.

39. Nawaz, Z., Patil, V., Paul, Y., Hegde, A. S., Arivazhagan, A., Santosh, V., et al. (2016) PI3 kinase pathway regulated miRNome in glioblastoma: identification of miR-326 as a tumour suppressor miRNA. Mol. Cancer 15; 74.

40. Ning, J., Zhang, H. and Yang, H. (2018) MicroRNA 326 inhibits endometrial fibrosis by regulating TGF $\beta 1 / \mathrm{Smad} 3$ pathway in intrauterine adhesions. Mol. Med. Rep. 18; 2286-2292.

41. Niwald, M., Migdalska-Sęk, M., Brzeziańska-Lasota, E. and Miller, E. (2017) Evaluation of Selected MicroRNAs Expression in Remission Phase of Multiple Sclerosis and Their Potential Link to Cognition, Depression, and Disability. J. Mol. Neurosci. $63 ; 275-282$.

42. Nye, J. S., Kopan, R. and Axel, R. (1994) An activated Notch suppresses neurogenesis and myogenesis but not gliogenesis in mammalian cells. Development 120; 2421-2430.

43. Ohl, K. and Tenbrock, K. (2015) Regulatory T cells in systemic 
lupus erythematosus. Eur. J. Immunol. 45; 344-355.

44. Pan, G., Mao, A., Liu, J., Lu, J., Ding, J. and Liu, W. (2020) Circular RNA hsa_circ_0061825 (circ-TFF1) contributes to breast cancer progression through targeting miR-326/TFF1 signalling. Cell Prolif. 53; e12720.

45. Pan, Y., Wan, J. and Wang, C. (2019) MiR-326: Promising Biomarker for Cancer. Cancer Manag. Res. 11; 10411-10418.

46. Pardini, B., De Maria, D., Francavilla, A., Di Gaetano, C., Ronco, G. and Naccarati, A. (2018) MicroRNAs as markers of progression in cervical cancer: a systematic review. BMC Cancer $18 ; 696$.

47. Ragusa, F., Fallahi, P., Elia, G., Gonnella, D., Paparo, S. R., Giusti, C., et al. (2019) Hashimotos' Thyroiditis: epidemiology, pathogenesis, clinic and therapy. Best Pract. Res. Clin. Endocrinol. Metab. 33; 101367.

48. Rak, K. and Bronkowska, M. (2018) Immunomodulatory effect of vitamin $\mathrm{D}$ and its potential role in the prevention and treatment of type 1 diabetes mellitus - A narrative review. Molecules 24; 53.

49. Rayman, M. P. (2019) Multiple nutritional factors and thyroid disease, with particular reference to autoimmune thyroid disease. Proc. Nutr. Soc. 78; 34-44.

50. Rose, N. R. (2016) Prediction and Prevention of Autoimmune Disease in the 21st Century: A Review and Preview. Am. J. Epidemiol. 183; 403-406.

51. Rupaimoole, R. and Slack, F. J. (2017) MicroRNA therapeutics: towards a new era for the management of cancer and other diseases. Nat. Rev. Drug Discov. 16; 203-222.

52. Sang-Ging, O., Won, H. L. and Kodo, K. (2015) MicroRNAmediated regulation of differentiation and trans-differentiation in stem cells. Adv. Drug Deliv. Rev. 88; 3-15.

53. Sebastiani, G., Grieco, F. A., Spagnuolo, I., Galleri, L., Cataldo, D. and Dotta, F. (2011) Increased expression of microRNA miR-326 in type 1 diabetic patients with ongoing islet autoimmunity. Diabetes Metab. Res. Rev. 27; 862-866.

54. Sonkoly, E. and Pivarcsi, A. (2009) microRNAs in Inflammation. Int. Rev. Immunol. 28; 535-561.

55. Sun, X., Tao, J., Xiang, N., Li, X., Wang, G., Fang, X., et al. (2016) Negative Correlation Between miR-326 and Ets-1 in Regulatory T Cells from new-Onset SLE Patients. Inflammation 39; 822-829.

56. Tang, Q., Chen, Z. and Zhao, L. (2019) Circular RNA hsa_circ_0000515 acts as a miR-326 sponge to promote cervical cancer progression through up-regulation of ELK1. Aging (Albany NY) 11; 9982-9999.

57. Vu, M., Yu, J., Awolude, O. A. and Chuang, L. (2018) Cervical Cancer Worldwide. Curr. Probl. Cancer 42; 457-465.

58. Waschbisch, A., Atiya, M., Linker, R. A., Potapov, S., Schwab,
S. and Derfuss, T. (2011) Glatiramer Acetate Treatment Normalizes Deregulated microRNA Expression in Relapsing Remitting Multiple Sclerosis. PLoS One 6; e24604.

59. Xia, Y., Tao, J., Fang, X., Dai, X., Xiang, N., Jin, L., et al. (2018) MicroRNA-326 Upregulates B Cell Activity and Autoantibody Production in Lupus Disease of MRL/lpr Mice. Mol. Ther. Nucleic Acids 11; 284-291.

60. Xu, T., Yan, W., Wu, Q., Xu, Q., Yuan, J., Li, Y., et al. (2019) MiR-326 Inhibits Inflammation and Promotes Autophagy in Silica-Induced Pulmonary Fibrosis through Targeting TNFSF14 and PTBP1. Chem. Res. Toxicol. 32; 2192-2203.

61. Xue, X., Chen, Q., Zhao, G., Zhao, J., Duan, Z. and Zheng, P. (2015) The Overexpression of TGF- $\beta$ and CCN2 in Intrauterine Adhesions Involves the NF-кB Signaling Pathway. PLoS One 10; 0146159 .

62. Yi, S. and Jin, Y. X. (2009) MicroRNA in cell differentiation and development. Sci China C Life Sci. 52; 205-211.

63. Yosef, N., Shalek, A. K., Gaublomme, J. T., Jin, H., Lee, Y., Awasthi, A., et al. (2013) Dynamic regulatory network controlling Th17 cell differentiation. Nature 496; 461-468.

64. Yu, D., Li, T., Xia, E., Huang, X., Liu, Y. and Peng, X. (2008) Factors affecting reproductive outcome of hysteroscopic adhesiolysis for Asherman's syndrome. Fertil. Steril. 89; 715-722.

65. Yu, S., Huang, H., Deng, G., Xie, Z., Ye, Y., Guo, R., et al. (2015) miR-326 Targets Antiapoptotic Bcl-xL and Mediates Apoptosis in Human Platelets. PLoS One 10; e0122784.

66. Zahednasab, H. and Balood, M. (2014) The role of miR-326 and miR-26a in MS disease activity. Gene 548; 158.

67. Zhang, P., Kong, F., Deng, X., Yu, Y., Hou, C. and Liang T. (2017) MicroRNA-326 suppresses the proliferation, migration and invasion of cervical cancer cells by targeting ELK1. Oncol. Lett. 13; 2949-2956.

68. Zhang, X., Olsen, N. and Zheng, S. (2020) The progress and prospect of regulatory $\mathrm{T}$ cells in autoimmune diseases. $J$. Autoimmun. 111; 102461.

69. Zhao, N., Zou, H., Qin, J., Fan, C., Liu, Y., Wang, S., et al. (2018) MicroRNA-326 contributes to autoimmune thyroiditis by targeting the Ets-1 protein. Endocrine 59; 120-129.

70. Zou, X., Liu, T., Gong, Z., Hu, C. and Zhang Z. (2017) MicroRNAs-mediated epithelial-mesenchymal transition in fibrotic diseases. Eur. J. Pharmacol. 796; 190-206.

This is an open access article distributed under the Creative Commons License (CC-BY-NC), which permits use, distribution and reproduction of the articles in any medium provided that the original work is properly cited and is not used for commercial purposes. 